\title{
A Real Virtual Pinhole
}

\author{
Ian Stephenson* \\ National Centre for Computer Animation \\ Bournemouth University
}

\begin{abstract}
Many publications on rendering at some point make reference to a theoretical or ideal pinhole camera model, the implication being that an infinitely small hole will produce a perfectly sharp image. However this is not the case. The assumptions of ray, and particle based models of light transport do not accurately represent the results obtained from small aperture systems. Here we consider a more appropriate model, which is capable of reproducing the results of a real pinhole camera, and in doing so generate an interesting interpretation of diffraction, as a sampling artifact.
\end{abstract}

\section{Diffraction Limiting}

Model of light transport based upon ray tracing indicate that a pinhole camera with a finite aperture will produce a blurred image. While real cameras require sufficient light to reach the film to produce an image within a practical length time, there is no such limitation within a simulation, and therefore the pinhole is typically reduced to a point, producing a perfectly sharp image.

However the implication that film speed is the only limitation upon the resolution of the pinhole camera is incorrect. While reducing the pinhole size will initially improve detail in the image, a certain point is reached (typically around $0.1 \mathrm{~mm}$ for cameras using visible light) beyond which reducing the pinhole causes more blurring in the image.

The problem is that for small apertures, a real pinhole camera is diffraction limited. Upon passing through the pinhole, the light will spread out - an effect that ray optics cannot simulate. While diffraction has little visible effect within typical scenes, the pinhole which sits at the center of so many rendering systems is one situation where the effect is particularly significant.

\section{Wave Representations}

In order to model diffraction we need to consider light as a series of waves. Each point within the pinhole receives light from visible surfaces. However unlike ray models, this light must be summed considering both its amplitude and phase. For the sake of tractability we consider only a single wavelength, allowing the resultant wave at each point to be represented by a single complex number.

The waves summed at all points which make up the pinhole fully capture the information about the scene which will then be transfered to the film. This is performed by treating each pinhole point as an emitter and summing them at all points on the film.

As a huge number of points must be evaluated within the pinhole to accurately capture the scenes light-field, this process is compute intensive. However the diffraction patterns produced by real pinholes are accurately reproduced. Optimal sharpness for point sources is produced when the simulation is of the dimensions suggested by theory. In more complex scenes unwanted interference can lead to noise, so the results of several simulations must be averaged.

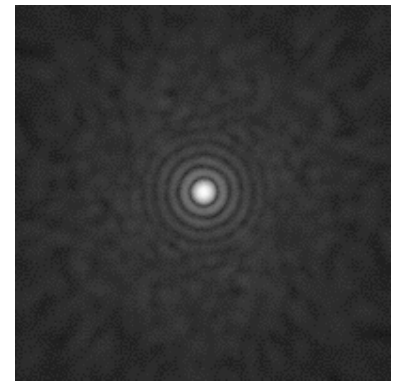

Diffraction of a Point Source

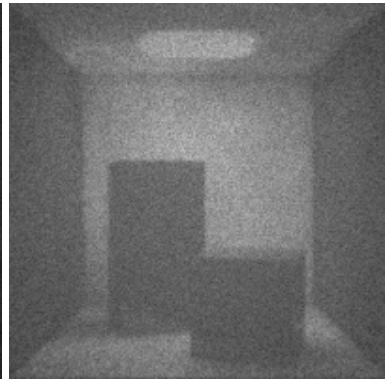

A Rendered Scene

\section{Diffraction as a Sampling Artifact}

To accurately capture the light within the scene at a particular wavelength we must sample the pinhole at intervals of $\lambda / 2$. For a pinhole of diameter $\mathrm{d}$ this produces $2 d / \lambda$ samples (considering only one dimension). While we may choose to increase the number of samples within our simulation to reduce sampling errors, this is simply a practical issue - the additional samples provide no new information. There can be no additional information in the final image which is not already captured in the pinhole samples, and hence $2 d / \lambda$ provides an upper bound upon the resolving power of our simulation, and in turn upon the pinhole camera.

When the pinhole is large, ray optics provide a second limit upon resolution: the image of the pinhole upon the film cannot be smaller than the diameter of the pinhole itself. For a film of width $D$ we therefore have a resolution limit of $D / d$. Optimal resolution is achieved when these two limits are equal: $D / d=2 d / \lambda$. We therefore derrive an optimal pinhole size of $d=\sqrt{D \lambda / 2}$

An alternative is to consider the optimal film size for a pinhole: $D=2 d^{2} / \lambda$. More conventional derivations based upon models of far-field diffraction propose an optimal distance from pinhole to film of: $f \approx 0.4 d^{2} / \lambda$. These results are equivalent if $D \approx 5 f:$ a camera geometry which is wider than normally used, but which would capture more of the information within the pinhole than a narrower field of view. Considering its simplicity, the model of diffraction limiting based upon Nyquist sampling agrees with the more complex optical models to a remarkably good degree of accuracy.

\section{Conclusion}

Ray optics cannot simulate all optical phenomena, and the pinhole camera is one such case. While wave optics are more powerful, they are more computationally intensive. Fortunately in the case of small apertures, simulation is possible. The results not only agree with those of real experiments, but the consideration of the pinhole camera as a sampling mechanism provides insight into the phenomenon of diffraction limiting — the pinhole is simply too small to squeeze the information through.

*e-mail: ian@dctsystems.co.uk 